\title{
A Survey on Hybrid Recommendation Systems
}

Prof. Pradnya Mehta ${ }^{1}$, Onkar Dongare ${ }^{2}$, Rushikesh Tekale ${ }^{2}$, Hitesh Umare'2, Rutwik Wanve²

${ }^{1}$ Assistant Professor, M.E. (Computer Engineering), Department of Computer Engineering, Marathwada Mitra

Mandal's College of Engineering, Pune, Maharashtra, India

${ }^{2}$ Student, Department of Computer Engineering, Marathwada Mitra Mandal's College of Engineering, Pune, Maharashtra, India

\section{Article Info}

Volume 7, Issue 6

Page Number: 219-224

Publication Issue :

November-December-2021

\section{Article History}

Accepted : 02 Dec 2021

Published : 10 Dec 2021

\section{ABSTRACT}

As India is moving fast towards digital economy, E-commerce industry has been on rise. Many platforms such as Amazon and Flipkart provide their customers with a shopping experience better than actual physical stores. Several Ecommerce websites use different methods to improve the customer engagement and revenue. One such technique is the use of personalized recommendation systems which uses customer's data like interests, purchase history, ratings to suggest new products which they may like. Recommendation systems are used by E-commerce websites to suggest new products to their users. The products can be suggested based on the top merchants on the website, based on the interests of the user or based the past purchase pattern of the customer. Recommender systems are machine learning based systems that help users discover new products. Due to the recent pandemic situation of 2020 and 2021, many of the local retail stores have been trying to shift their business to online platforms such as dedicated websites or social media. The proposed methodology based on Machine Learning aims to enable local online retail business owners to enhance their customer engagement and revenue by providing users with personalized recommendations using past data using methods such as Collaborative Filtering and Content-Based Filtering.

Keywords : E-commerce, Recommendation Systems (RS), Machine Learning, Personalized recommendations, Collaborative Filtering, Content Based Filtering, TF-IDF, Popularity Based Recommendation System

\section{INTRODUCTION}

Every time we visit any shopping website such as Amazon, or a music or video streaming site such as Spotify or Youtube, we are greeted with new products that we do not realize we want until we see them.
Whenever we view a product or rate any previously bought product, the next time we visit the website, we are presented with products that are very similar to what we have previously bought or new products that other users with same taste have bought. This is made possible using Machine Learning based 
recommendation systems that learn from our past behavior and predict or future behavior. These systems are used by almost every E-commerce giants to basically attract their customers back to them. Unlike traditional commerce that is carried out physically by a person to sell items and inform customers about new products, E-commerce and recommendation systems have made is easier for us to buy products as well as discover new ones.

These systems work by analysing our behaviour such as ratings and preferences and use these attributes to predict which products we may like. These systems are mostly built using two techniques called collaborative filtering and content based filtering. The collaborative filtering approach works by recommending products that other users have bought and the content based filtering method works by recommending products having similar attributes to the user's previous purchases.

However, the cost of developing and deploying such systems is huge and certainly cannot be afforded by every local online business owner.

Collaborative filtering method comprises of two subtypes called user-user collaborative filtering and item-item collaborative filtering. The user-user collaborative filtering takes other users into consideration while calculating the similarity and then recommends the products bought by them to the active user. Here, the correlation between the different users is identified. The item-item collaborative filtering works by considering the correlation between items, or in this case, products. If an item is similar to another item, it is recommended. The only requirement here is the availability of sufficient data. This method requires dataset consisting of the product name and/or id, the user Id and the rating for that product. Based on this data we can successfully perform collaborative filtering. We also have to address the incomplete or unavailable data in our dataset as it can have a negative effect on the performance of the algorithm. The data is then transformed into a matrix of $\mathrm{n} \mathrm{x} \mathrm{m}$ in which the similarities between the users or the items is identified depending in the method of choice. The higher the similarity, the greater is the probability of the user liking that product.

However, the Content-Based Filtering approach works differently. It attempts to determine what an user's favourite aspect of an item is and then recommends items that present those aspects. The aspect can be anything like genre of a movie, colour of a clothing item. It then ranks similar products corresponding to their similarity scores and recommends the most similar products to the user. This method also uses a technique named Term Frequency and Inverse Document Frequency commonly mentioned as TF-IDF [4] which calculates the recurrence of a term in a document and the total number of documents which include the term respectively. Another type of such system is the Popularity-Based Recommendation System. These type of systems works on the basis of popularity of an item. Any item which is most popular and trending among the users is directly recommended to the active user.

Instead of using either one of these methods to provide personalized recommendations to the users, combining all of these into a Hybrid Recommendation System is the best option. Using only either of the method such as collaborative filtering can give rise to an issue known as cold-start, where a new user cannot get any recommendations from the system due to lack of any previous data. In such cases, collaborative filtering paired with content-based filtering and/or popularity based model can be the solution. 
Prof. Pradnya Mehta et al Int. J. Sci. Res. Comput. Sci. Eng. Inf. Technol, November-December-2021, 7 (6) : $219-224$

\section{LITERATURE REVIEW}

An exploratory research was conducted which applied and demonstrated the method of data mining with the help of association rule technique and itembased collaborative filtering. It was used in an approach for baseline recommender systems in ecommerce. It uses the review dataset from Amazon from the year 2018 and focuses on online shopping of phones and their accessories. [1]

Investigation of accuracy issues of implicit feedback in a RS was done and a system developed on hybrid Real Time Incremental Stochastic Gradient Descent was presented by combining Alternating Least Squares with Weight Regularization and SGD. This helped for faster updating of the martix factorization model. [2]

The system uses dataset from Movie-Lens and constructs a matrix of users and ratings. It uses a deep learning approach to calculate the similarity between the items. It then uses matrix factorization to generate the recommendations. The method shows that, involvement with datasets can convey exactness better than traditional closest neighbor approach. [3]

TF-IDF i.e. Term Frequency and Inverse Document Frequency which identifies the occurrence of a term in a document and the total number of documents containing the term respectively can be used to generate product tags based on the product description. By doing so, the process becomes quite efficient and dynamic. [4]

Flipkart Product Recommendation System is built on Content Based, Collaborative Based and Hybrid Filtering Techniques to attain accurate recommendations. Content based Filtering uses the history of purchase of the active user to evaluate proper recommendations. Collaborative Filtering is a prediction technique that does not depend on the domain. It matches the users which have similar preferences. Collaborative Filtering is further classified into Item Based Filtering and User Based Filtering. [5]

Collaborative Filtering and Deep Learning based RS addresses the problem for CSS and ICS items. It uses the architecture SDAE which is based on deep learning. This system uses two types of methods: Collaborative Filtering is a prediction technique that looks for the users which have similar interests. Deep Learning uses datasets from reputed sources containing millions of reviews about a product. There are two classifications of feedbacks: Implicit Feedbacks and Explicit Feedbacks. Implicit Feedbacks are the indirect feedbacks taken from a user for example when a user watches a youtube video. Most of new recommendation systems use implicit feedback. Explicit Feedbacks are the direct feedbacks taken from the user like the rating system for products on amazon's website. This System with these two techniques combined is effective to handle Cold Start Problem. [6]

The Book Recommender System has considered many several attributes like category of the book and also content by applying techniques such as collaborative filtering to the ratings given by the other users. This recommender system also utilizes an association based model to give accurate recommendations. It has offline recommendations so the performance is not affected. [7]

Automated Web Usage Data Mining and Recommendation System uses K-Nearest Neighbor $(\mathrm{KNN})$ method because it is one of the simplest problem solving method. Unlike many existing data mining methods, it overcomes the problem of Scalability, reduces Error rate and provides more accurate and faster recommendations. The system has provided a base for Real-Time recommendation systems. It collects "click stream" and "matches" on active sessions from the users and compares them 
with dataset in the data mart to generate such accurate recommendations in real time. [8]

State of the Art Approach is categorized into four types of approaches. They are Content Based Filtering, Collaborative Based Filtering, Demographic Filtering and Hybrid Filtering. It also manifests the ranking (rating) of a Product. Ranking maximizes the user convenience. Thus, rank plays an important role in the recommendation systems. It ensures the optimal ordering of a group of items for the user. [9]

Recommender System Application Development analyzes traditional techniques fundamentally Content Based System, Collaborative System, Knowledge Based System and Hybrid Methods and also latest techniques like Fuzzy set based, Social Network based, Trust based, Content Awareness based and Group based. This system also introduces techniques such as E-Government recommender system, E-Business Recommender System, ECommerce Recommender System, Tourism Recommender System, TV Program based Recommender System and Group Activity Recommender System. [10]

Recommender System traverses through various prediction techniques and analyzes the characteristics and potential of these systems and guides the field of recommendation system in the proper direction. It explores the various phases of a recommender system like Information Gathering Phase, Explicit Feedback, Implicit Feedback, Hybrid Feedback, Learning Feedback, Prediction Phase, Weighted Hybridization, Switching Hybridization and Cascade Hybridization. Also, this paper puts a light on Advantages and Limitations of these Hybridization techniques. The knowledge secured during this study helps the researchers to enhance the road map of State of the Art Recommendation systems. [11]
Product Recommendation System will require large quantity of data in order to arrive at the correct decision. The information which is provided to the recommender system must be persistent in nature. For the method, some sort of preset information is required. The recommender system gathers the information and calculates the decision in two possible ways by the use of Collaborative Filtering or by the use of Content Based Filtering. The collaborative filtering is the method of filtering for data among the multiple sources. On the other hand Content Based Filtering will filter the data which is going to be used within the system. Content filtering will be utilized here. The users have preferences for the certain items. The preferences of the users continuously change because of which recommendation system is created. [12]

Empirical Analysis of the Impact of Recommendation System interrogates a primary question that is the influence of these systems on sales. Considering the interaction between sales, recommendations, and the retail process, a robust model was developed that comprises of the indirect effect of recommendations on potential coexistence between sales and recommendations, and sales through retailer pricing, and an overall calculation of the strength of recommendations. During a survey on two online retailers, they came to the conclusion that the Recommendations have positive impact not only on sales but also on price of the product by providing the flexibility to adjust it. Analysis has revealed that recommendations have shown better results in increasing the sales even greater than customer feedback. [13]

A Literature Survey on Recommendations Systems based on Sentimental Analysis digs into various techniques such as the weighted algorithms which are used to produce scores for multiple texts. The methodology of sentiment analysis covers the way for the advancement of recommender system on personal 
Prof. Pradnya Mehta et al Int. J. Sci. Res. Comput. Sci. Eng. Inf. Technol, November-December-2021, 7 (6) : 219-224

basis. Substantial research has been done on recommendation systems and they have been mainly divided into three categories: Collaborative filtering, Content Based and Context Based recommendation systems. The research shows the employment of Sentimental Analysis in developing of a more accurate Recommender System. [14]

Convolutional Neural Networks can also be used for recommender system. CNN is a type of neural network that is used for classification purposes. For collaborative filtering, user id and product id can be given as inputs to the network and can be used to extract features. These features are then assigned weights. CNNs include various applications including Soil Nutrients and Leaf Disease Detection. [15]

\section{III.CONCLUSION}

We will be able to build a hybrid recommendation system comprising of different algorithms which will predict the items that may be preferred by the user. This system shall be made completely free to use and deploy to anyone who wishes to add it to their system. This methodology shall allow local online retail business owners to directly deploy the recommendation system into their existing system thus generating more revenue and attracting more customers.

\section{REFERENCES}

[1]. Jessica Lourenco and Aparna S. Varde, "ItemBased Collaborative Filtering and Association Rules for a Baseline Recommender in ECommerce", 20th IEEE International Conference on Big Data, 2020. DOI:10.1109/BigData50022.2020.9377807.

[2]. C. Lin, L. Wang and K. Tsai, "Hybrid Real-Time Matrix Factorization for Implicit Feedback Recommendation Sys-tems", IEEE Access, vol.
6, $\quad$ pp. 21369-21380, 2018. DOI:10.1109/ACCESS.2018.2819428

[3]. Rahul Katarya, DTU, “An Improved Recommender Sys-tem Using Two-Level Matrix Factorization for Product On-tologies", International Conference on Intelligent Sustaina-ble Systems, 2017. DOI:10.1109/ISS1.2017.8389402

[4]. Tessy Badriyah, Erry Tri Wijayanto, Iwan Syarif, Prima Kristalina, "A Hybrid Recommendation System for E-Commerce based on Product Description and User Profile", The Seventh International Conference on Innovative Com-puting Technology (INTECH), 2017 DOI:10.1109/INTECH.2017.8102435

[5]. T. Keerthana, T. Bhavani, N. Suma Priya, V. Sai Prathyusha, K.Santhi Sri (2020), "Flipkart Product Recom-mendation System", Journal of Engineering Sciences, Vol. 11, Issue 4, April 2020. DOI:10.15433.JES.2020.V11I04.43P.70

[6]. Jian Wei, Jianhua He, Kai Chen, Yi Zhou, Zuoyin Tang, "Collaborative filtering and deep learning based recommen-dation system for cold start items", Expert Systems with Applications, Elsvier, Volume 69, 2017, Pages 2939 , ISSN 0957-4174 https://doi.org/10.1016/j.eswa.2016.09.040.

[7]. Ms. Sushama Rajpurkar, Ms. Darshana Bhatt, Ms. Pooja Malhotra, "Book Recommendation System”, JIRST -International Journal for Innovative Research in Science Technology Volume 1, Issue 11, April 2015, ISSN: 23496010.

[8]. D.A. Adeniyi, Z. Wei, Y. Yongquan, "Automated web usage data mining and recommendation system using K-Nearest Neighbor (KNN) classification method", Applied Computing and Informatics, Volume 12, Issue 1, 2016, ISSN2210-8327. https://doi.org/10.1016/j.aci.2014.10.001

[9]. Mohammad Aamir, Mamta Bhusry, "Recommendation System: State of the Art 
Approach", JIRST -International Journal for Innovative Research in Science Technology, Volume 1, Issue 11, April 2015, ISSN: 23496010 .

[10]. Jie Lu, Dianshuang Wu, Mingsong Mao, Wei Wang, Guangquan Zhang, "Recommender system application de-velopments: A survey", Decision Support Systems, Elsvier, Volume 74, 2015, Pages 12-32, ISSN 0167-9236. https://doi.org/10.1016/j.dss.2015.03.008

[11]. F.O. Isinkaye, Y.O. Folajimi, B.A. Ojokoh, "Recom-mendation systems: Principles, methods and evaluation", Egyptian Informatics Journal, Volume 16, Issue 3, 2015, Pages 261273, ISSN 1110-8665. https://doi.org/10.1016/j.eij.2015.06.005

[12]. Jatinder Kaur, Rajeev Kumar Bedi, S.K. Gupta "Prod-uct Recommendation Systems a Comprehensive Review." International Journal of Computer Sciences and Engineer-ing 6.6 (2018), 1192-1195.

[13]. Bhavik Pathak, Robert Garfinkel, Ram D. Gopal, Raj-kumar Venkatesan Fang Yin, "Empirical Analysis of the Impact of Recommender Systems on Sales", Journal of Management Information Systems, Volume 27, 2010, Pages 159-188. https://doi.org/10.2753/MIS0742-1222270205

[14]. Achin Jain, Vanita Jain and Nidhi Kapoor, "A Literature Survey on Recommendation System based on Sentimental Analysis", Advanced Computational Intelligence Journal, Vol. 3, No.1, January 2016.

[15]. Ms. Pradnya Samit Mehta, Ms. Sarita DnyaneshwarSapkal, Ms. Geeta Chillarge, "Smart Cultivation and Prediction System for Agriculture", International Jour-nal of Grid and Distributed Computing, Vol 13, No.2, 2020.

\section{Cite this article as :}

Prof. Pradnya Mehta, Onkar Dongare, Rushikesh Tekale, Hitesh Umare, Rutwik Wanve, "A Survey on Hybrid Recommendation Systems", International Journal of Scientific Research in Computer Science, Engineering and Information Technology (IJSRCSEIT), ISSN : 2456-3307, Volume 7 Issue 6, pp. 219-224, November-December 2021. Available at doi : https://doi.org/10.32628/CSEIT217628 Journal URL : https://ijsrcseit.com/CSEIT217628

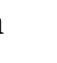

